OPEN ACCESS

Edited by:

Valérie Doyère,

Centre National de la Recherche

Scientifique, France

Reviewed by:

Etienne Coutureau,

Centre National de la Recherche Scientifique/Université de Bordeaux,

France

Jeremy J. Clark

University of Washington, USA

*Correspondence:

Alicia Izquierdo,

Department of Psychology, University of California at Los Angeles, 1285 Franz Hall Box 951563 Los Angeles, CA 90095-1563, USA aizquie@psych.ucla.edu

Received: 09 March 2015 Accepted: 21 April 2015 Published: 07 May 2015

Citation:

Stolyarova A and lzquierdo A (2015) Distinct patterns of outcome valuation and amygdala-prefrontal cortex synaptic remodeling in adolescence and adulthood. Front. Behav. Neurosci. 9:115. doi: 10.3389/fnbeh.2015.00115

\section{Distinct patterns of outcome valuation and amygdala-prefrontal cortex synaptic remodeling in adolescence and adulthood}

\author{
Alexandra Stolyarova ${ }^{1,2}$ and Alicia Izquierdo 1,2* \\ ${ }^{1}$ Department of Psychology, University of California at Los Angeles, Los Angeles, CA, USA, ${ }^{2}$ Brain Research Institute, \\ University of California at Los Angeles, Los Angeles, CA, USA
}

Adolescent behavior is typified by increased risk-taking, reward- and novelty-seeking, as well as an augmented need for social and environmental stimulation. This behavioral phenotype may result from alterations in outcome valuation or reward learning. In the present set of experiments, we directly compared adult and adolescent animals on tasks measuring both of these processes. Additionally, we examined developmental differences in dopamine D1-like receptor (D1R), dopamine D2-like receptor (D2R), and polysialylated neural cell adhesion molecule (PSA-NCAM) expression in animals that were trained on an effortful reward valuation task, given that these proteins play an important role in the functional development of the amygdala-prefrontocortical (PFC) circuit and mesocorticolimbic dopamine system. We found that adolescent animals were not different from adults in appetitive associative learning, but exhibited distinct pattern of responses to differences in outcome values, which was paralleled by an enhanced motivation to invest effort to obtain larger rewards. There were no differences in D2 receptor expression, but D1 receptor expression was significantly reduced in the striatum of animals that had experiences with reward learning during adolescence compared to animals that went through the same experiences in adulthood. We observed increased levels of PSA-NCAM expression in both PFC and amygdala of late adolescents compared to adults that were previously trained on an effortful reward valuation task. PSA-NCAM levels in PFC were strongly and positively associated with high effort/reward (HER) choices in adolescents, but not in adult animals. Increased levels of PSA-NCAM expression in adolescents may index increased structural plasticity and represent a neural correlate of a reward sensitive endophenotype.

Keywords: D1 receptors, D2 receptors, PSA-NCAM, stimulus-reward, effort, decision-making, cost-benefit

\section{Introduction}

Adolescence is a critical period during which animals learn to predict future states of their habitat depending on current experiences and acquire life strategies that are likely to promote survival and reproductive success later in life. The fitness is increased if the phenotype that developed in early life is matched to the predicted environment (Gluckman et al., 2007), and if an animal can adequately cope with the environmental uncertainty and reward availability 
(McNamara et al., 2013). Altricial rodents venture out of their home burrow at Post Natal Day (PND) 28, leaving the care of their adult conspecifics, and learn how to acquire nutrients and safety independently (Galef, 1981). The adolescent (PND 28-60) behavioral profile is characterized by increased risktaking, reward- and novelty-seeking, as well as an augmented need for social and environmental stimulation (Laviola et al., 2003; Kelley et al., 2004; Marco et al., 2011) that may have evolved to promote attainment of the necessary skills for independence (Spear, 2000).

Some of the behavioral patterns common to adolescents across species may result from alterations in reward valuation, marked by an increased sensitivity to reinforcers and reduced sensitivity to costs associated with obtaining them, or stimulusreward association learning. From a neurodevelopmental perspective, the adolescent period is characterized by pronounced changes in the functional organization and connectivity of the amygdala-prefrontal cortex (PFC) circuit (Cunningham et al., 2002, 2008) and mesocorticolimbic dopamine system (Gelbard et al., 1989; Tarazi and Baldessarini, 2000). Dopaminergic neurotransmission within striatum and PFC is critical to adaptive reward learning and motivation (Berridge and Robinson, 1998; Salamone and Correa, 2002; Cagniard et al., 2006; Ostlund et al., 2011; Salamone et al., 2012; Richard et al., 2013). D1-like (D1R) receptor signaling contributes to cortico-striatal plasticity and regulates reward learning and effort-based decision making (Beninger and Miller, 1998; Baldwin et al., 2002; Schweimer and Hauber, 2006). Similarly, D2-like (D2R) receptor-mediated signaling in striatum has been linked to effort expenditure toward palatable rewards (Trifilieff et al., 2013) and learning from positive outcomes (Groman et al., 2011). The adolescent period is marked by extensive pruning of dopamine D1R and D2R in the striatum (Gelbard et al., 1989; Teicher et al., 1995; Tarazi and Baldessarini, 2000) that may be associated with behavioral differences in reward choices in adolescent vs. adult animals.

Connections between amygdala and PFC are critical for reward responses and choosing between options of different value (Baxter et al., 2000; Blair et al., 2006; Waraczynski, 2006). Structural remodeling within this circuit may be partially dependent on neural cell adhesion molecule (NCAM) function. Previous research has shown that polysialylated NCAM (PSANCAM) is critical in synaptic remodeling and plasticity (Muller et al., 1996; Durbec and Cremer, 2001) and modulates cortical neuron sensitivity to neurotrophins (Vutskits et al., 2001). It is expressed in brain regions undergoing structural reorganization during development and in adulthood, including hippocampus, amygdala, and PFC (Nacher et al., 2002a,b; Seki, 2002; Varea et al., 2005). Interestingly, dopamine signaling and PSA-NCAM expression show bidirectional interactions: manipulations of dopamine signaling (systemically and in medial PFC) has been linked to alterations in PSA-NCAM expression (CastilloGómez et al., 2008), and a role for PSA-NCAM in dopamine signaling-induced plasticity of PFC inhibitory circuits has also been suggested (Nacher et al., 2013). Similarly, NCAM can promote $\mathrm{D} 2 \mathrm{R}$ internalization and subsequent degradation as well as modulate receptor-mediated signaling and behavior (Xiao et al., 2009). PSA-NCAM has already been implicated in learning and stress responses (Pham et al., 2003; Cordero et al., 2005; Bisaz et al., 2009). However, most of the work to date has focused on its role in aversive learning and fear memory, and largely centered on hippocampal function (Senkov et al., 2006; Lopez-Fernandez et al., 2007; Kochlamazashvili et al., 2010). It is not known if PSA-NCAM also contributes to appetitive responses and if the regional specificity of its expression is developmentally specific. This molecular target is of a particular interest as NCAM polysialylation has been linked to neurodevelopmental predisposition to schizophrenia (Hildebrandt et al., 2009), abnormal social interaction and aggression (Calandreau et al., 2010), as well as individual risk for alcohol-related behaviors (Barker et al., 2012). Therefore, in the present set of experiments, we directly compared adult and adolescent animals on tasks measuring both stimulusreward association learning (Experiment 1) and reward valuation (Experiment 2). Additionally, in Experiment 3, we examined developmental differences in dopamine D1R and D2R expression in striatum and PFC as well as PSA-NCAM expression in PFC and amygdala in adolescent and adult animals trained on an effortful reward valuation task (Stolyarova et al., 2015).

\section{Material and Methods}

\section{Subjects}

Subjects were 24 (Adult $=12$, Adolescent $=12$ ) male Long Evans rats (Charles River Laboratories), pair housed. Adolescent animals arrived at our facility at PND 25. They were PND 28 and adult animals were PND 65 at the beginning of handling. Vivaria were maintained under a $12 / 12 \mathrm{~h}$ light/dark cycle at $22^{\circ} \mathrm{C}$. All behavioral testing took place 5-7 days a week between 08:00 and 16:00 h during the rats' inactive period, consistent with previous and ongoing studies in our lab. Research protocols were approved by the Chancellor's Animal Research Committee at the University of California, Los Angeles.

\section{Handling and Food Restriction}

Rats were left undisturbed for 3 days after arrival to our facility to acclimate to the vivarium. Each rat was then handled for a minimum of $10 \mathrm{~min}$ once per day for 5 days. Animals were foodrestricted to ensure motivation to work for food for a week prior and during the behavioral testing, while water was available ad libitum. All rats were food restricted based on their baseline food intake that was assessed after the animals had already acclimated to the vivarium to control for the effects of stress on feeding behavior. Food availability was gradually decreased starting with $80 \%$ of baseline intake. The amount of food given was never lower than $50 \%$ baseline. Weights were monitored daily. We ensured that adult animals did not fall below $85 \%$ of their freefeeding body weight and adolescent animals fell within normal age-matched growth weights provided by the vendor. On the two last days of food restriction prior to behavioral training, rats were fed twenty $\frac{1}{2}$ froot loops or sugar pellets in their home cage to accustom them to the food rewards. 


\section{Experiment 1. Reward Learning in Adolescent and Adult Animals}

\section{Behavioral Apparatus}

Behavioral testing was conducted in operant conditioning chambers (Model 80604 Lafayette Instrument Co., Lafayette, IN) that were housed within sound- and light- attenuating cubicles. Each chamber was equipped with a house light, tone generator, video camera, and LCD touchscreen opposing the pellet dispenser. The pellet dispenser delivered 45-mg dustless precision sucrose pellets. Software (ABET II TOUCH) controlled touchscreen stimuli presentation, tone generation, tray- and house-light illumination and pellet dispensation.

\section{Behavioral Training}

Reward learning was assessed on tasks commonly used as pretraining stages for discrimination learning testing. The training protocol was adapted from Kosheleff et al. (2012) and Izquierdo et al. (2010). Due to a short duration of adolescence in rats (i.e., PND 28-60), only three initial phases were used in the present experiment: Habituation, Initial Touch Training (ITT), and Must Touch Training (MTT). During habituation, rats were required to eat five pellets out of the pellet dispenser inside of the chambers within 15 min before exposure to any stimuli on the touchscreen. ITT began with the display of white graphic stimuli on the black background at the bottom of the touchscreen. The stimuli measured $45 \times 45 \mathrm{~mm}^{2}$ and were within reach for both adult and adolescent animals. During this stage a trial could be terminated for one of two reasons: if a rat touched the displayed stimulus, or if the stimulus display time (40 s) ended, after which the stimulus was removed and black background displayed. The disappearance of the image was paired with the onset of a "reinforcer event": dispensation of one (low reward, LR; at the termination of stimulus time) or three (high reward, HR; stimulus touched) sucrose pellets, a 1 stone, and an illumination of the tray-light. Trials were separated by 10 s ITI. In MTT, a trial could be terminated only if the rat touched the image, which then disappeared followed by reward delivery. For both ITT and MTT, the criterion for advancement was set to 60 rewards consumed in $45 \mathrm{~min}$. Animals were given one $45 \mathrm{~min}$ session per day until the criterion was reached.

\section{Experiment 2. Outcome Valuation in Adolescent and Adult Animals \\ Behavioral Testing Apparatus}

Rats were tested on a task previously described in Stolyarova et al. (2015) which utilized a maze with three possible courses of action, each associated with different effort requirements and reward magnitudes. Behavioral training and testing were conducted in a standard eight-arm radial maze with arms extending from a central arena with a diameter of $25 \mathrm{~cm}$. Arms were $50 \mathrm{~cm}$ long and $12 \mathrm{~cm}$ wide. The positions of extramaze cues remained constant throughout all phases of the experiment. The four arms nearest the start arm were permanently blocked, leaving a start arm and three choice arms accessible to animals. One arm of the maze was randomly designated as a low effort/reward (LER), another as a medium effort/reward (MER), and the third as a high effort/reward (HER) arm. The arm assignment was counterbalanced across animals, and held constant between sessions. The barrier heights associated with MER and HER options were adjusted for the present experiment compared to previous study due to reduced ability of adolescent animals to climb over the tallest $30 \mathrm{~cm}$ barrier. The arm containing the low reward was unimpeded by a barrier, but in order to obtain the medium or high reward, rats were required to climb a $15 \mathrm{~cm}$ or $25 \mathrm{~cm}$ barrier, respectively. Rats were required to climb straight up the side $\left(90^{\circ}\right)$ and down at an angle to the food reward located at the end of the goal arm. "Froot loops" (Kellogg NA Co., Battle Creek, MI) were given as food rewards during testing: a "high reward" consisted of four $\frac{1}{2}$ froot loops (i.e., two froot loops), a "medium reward" consisted of two $\frac{1}{2}$ froot loops ( 1 froot loop), and a "low reward" consisted of one $\frac{1}{2}$ froot loop. Between trials, the rat was removed from the maze and placed in clear Plexiglas holding chamber with a $1651 \mathrm{~cm}^{2}$ base and $38.1 \mathrm{~cm}$ walls.

\section{Habituation}

A habituation and training protocol adapted from Walton et al. (2002) was used to habituate the rats to the maze and familiarize them with the froot loops. During the acclimation phase $5 \frac{1}{2}$ froot loops were placed into each arm of the maze (20 total). Each rat was individually placed into the maze and allowed to explore and eat froot loops freely. Criterion for advancement to the next phase was consumption of $20 \frac{1}{2}$ froot loops within $15 \mathrm{~min}$.

\section{Reward Magnitude Training. Phase 1}

In this phase, one goal arm was baited with four $\frac{1}{2}$ froot loops (HR arm), another with two $\frac{1}{2}$ froot loops (MR arm), and the third arm with one $\frac{1}{2}$ froot loop (LR arm). The arm assignment was counterbalanced across animals, and held constant between sessions. Rats were allowed to sample freely from all arms for ten trials. No barriers were present at this phase. Each trial lasted until the rat finished all the froot loops. Trials were separated by a $30 \mathrm{~s}$ inter-trial interval (ITI), during which time they were placed in an empty holding chamber. The order of arm visits was recorded. Criterion for advancement to the next phase was completion of ten trials within $30 \mathrm{~min}$.

\section{Reward Magnitude Training. Phase 2}

This phase was similar to Phase 1 of reward magnitude training, except that animals were allowed to visit only one arm per trial. Rats were removed from the maze as soon as the arm was chosen and the reward was consumed. Animals were given 10 trials per day separated by a $30 \mathrm{~s}$ ITI. This phase marked the beginning of learning to visit only one arm as well as continuing to learn each arm's associated reward values. Criterion for advancement to the next phase was choice of HR arm on $80 \%$ or more of the trials for two consecutive days.

\section{Alternating Free/Forced Choice Trials with Barriers}

During this phase, rats were required to climb barriers to achieve higher rewards. The LER arm continued to be unimpeded by 
a barrier, but in order to obtain the medium (MER) or high (HER) reward, rats were required to climb a $15 \mathrm{~cm}$ or $25 \mathrm{~cm}$ barrier, respectively. Thirteen trials were administered per day. Each day of testing consisted of ten free and three forced choice (one for each arm) trials, administered at the beginning. Thus, the structure of the testing was as follows: forced choice trials (1 through 3 ), followed by ten free choice trials (4 through 13). On forced choice trials all goal arms except one were blocked. The order of arm presentation during forced choice trials was counterbalanced between days. Upon eating the food reward, the rat was placed in a holding chamber for a $30 \mathrm{~s}$ ITI, during which the maze was wiped clean with $70 \%$ ethanol to prevent the rat's use of scent-guided choice. Rats were tested daily until stable baseline choice performance was established (choice preferences on free choice trials did not differ across three consecutive days).

\section{Experiment 3. Amygdala and PFC PSA-NCAM and Striatal D1R and D2R Expression in Adolescent and Adult Animals} Tissue Dissection

Rats from Experiment 2 were euthanized 1d after the last day of behavioral testing (late adolescent, PND 50 = 8; adult, PND $86=8)$ with an overdose of sodium pentobarbital $(250 \mathrm{mg} / \mathrm{kg}$, i.p.) and decapitated. The brains were immediately extracted and two millimeter-thick coronal sections of frontal cortex, striatum, and amygdala were further rapidly dissected, using a brain matrix, over wet ice at $4^{\circ} \mathrm{C}$. Frontocortical dissections included ventral (orbital) and medial sectors of the frontal cortex, but excluded most lateral, posterior (agranular insular) regions. Striatal dissections included both dorsal and ventral subregions.

\section{ELISA Method}

To prepare the tissues for the assays $0.3 \mathrm{~mL}$ (frontal cortex, striatum) or $0.2 \mathrm{~mL}$ (amygdala) of PBS $(0.01 \mathrm{~mol} / \mathrm{L}$, $\mathrm{pH}$ 7.2) containing a protease and phosphatase inhibitor cocktail (aprotinin, bestatin, E-64; leupeptin, NaF, sodium orthovanadate, sodium pyrophosphate, $\beta$-glycerophosphate; Thermo Scientific, Rockford, IL) was added to each sample. Each tissue was minced, homogenized, sonicated with an ultrasonic cell disrupter, and centrifuged at 5,000 g at $4^{\circ} \mathrm{C}$ for $10 \mathrm{~min}$. Supernatants were removed and stored at $-20^{\circ} \mathrm{C}$ until ELISA assays were performed. Bradford protein assays were also performed to determine total protein concentrations in each sample. D1R, D2R (Cat\# SEB299Ra and SEA673Ra, Cloud-Clone Corp., Houston, TX) and PSA-NCAM (Cat\# 67-ABC0027B, ALPCO Diagnostics, Salem, NH) protein levels were determined using a commercially-available ELISA kits. The assays were performed according to the manufacturer's instructions. The sensitivity of the assays is $0.055 \mathrm{ng} / \mathrm{mL}$ for D1R, $0.112 \mathrm{ng} / \mathrm{mL}$ for $\mathrm{D} 2 \mathrm{R}$, and $0.25 \mathrm{ng} / \mathrm{mL}$ for PSA-NCAM, and the detection range is $0.156-10 \mathrm{ng} / \mathrm{mL}$ for $\mathrm{D} 1 \mathrm{R}, 0.312-20 \mathrm{ng} / \mathrm{mL}$ for $\mathrm{D} 2 \mathrm{R}$, and $0.25-16 \mathrm{ng} / \mathrm{mL}$ for PSA-NCAM. The concentration of each protein is presented as ng/mg of total protein accounting for dilution factor.

\section{Data Analyses}

Software package SPSS (SAS Institute, Inc., Version 16.0) was used for statistical analyses. Statistical significance was noted when $p$ values less than 0.05 , and a trend towards significance was noted when $p$ values were $0.06-0.08$. Days to complete pre-training and establish stable performance were analyzed using $t$-tests. ITT and MIT learning and latency data were analyzed with independent sample $t$-tests and repeatedmeasures ANOVA (rmANOVA) with trial type as withinand developmental group as between-subject factors where appropriate. The maze choice data were first analyzed using multivariate ANOVA (MANOVA) with barrier height (LER, MER, HER) as within-subject and age group as between-subject factors to probe for differences in choice preferences. When significant interactions were found, post hoc simple main effects were reported. ELISA data were analyzed with independent samples $t$-tests.

\section{Results}

\section{Adolescent Animals Showed Normal Pattern of Growth and Development}

To ensure that the mild food restriction did not compromise healthy growth and development of the animals, we analyzed the pattern of weight fluctuations. Although there was an initial weight loss in the adult group (average maximal weight loss $=11 \%$ of baseline), both age groups showed an increase in weight by the end of the study (Figure 1A; main effect of time: $\left.F_{(18,396)}=30.843, p<0.001\right)$, which likely resulted from the supplemental nutrition obtained from the rewards earned during testing. As expected, the average weight gain in adolescent animals was higher than in adults (Figure 1B; $t_{(22)}=6.82, p<$ 0.0001).

\section{Experiment 1. Adolescent Animals are Indistinguishable from Adults in Reward Learning, but Show Distinct Pattern of Responses to Differences in Outcome Values}

All animals readily completed ITT and MTT. ITT criterion was reached in one session by the majority of the animals, whereas the range for MTT completion was 1-3 sessions. Adolescent and adult animals mastered the task at a comparable rate, there were no group differences in sessions to criterion on either of the tasks (MTT: $t_{(6)}=0.361, p=0.73$ ), or percent correct in ITT task $\left(t_{(6)}=0.678, p=0.523\right)$, suggesting that adolescent animals are not different from adults in acquiring stimulus-reward contingencies. To verify that the apparent lack of differences in learning rates between age groups were not due to ceiling effects, we analyzed learning progression within session by comparing performance in blocks of 10 trials. All animals improved their performance within session (Figure 2A; main effect of trial block: $\left.F_{(5,30)}=24.781, p<0.0001\right)$ with no differences between age groups: no main effect of age $\left(F_{(1,6)}=0.249, p=0.635\right)$ or age $\times$ trial block interaction $\left(F_{(5,30)}=0.159, p=0.75\right)$. To establish that animals learned the contingency of reward on the stimuli, we also analyzed animals' responses during the ITI intervals. All animals 
A

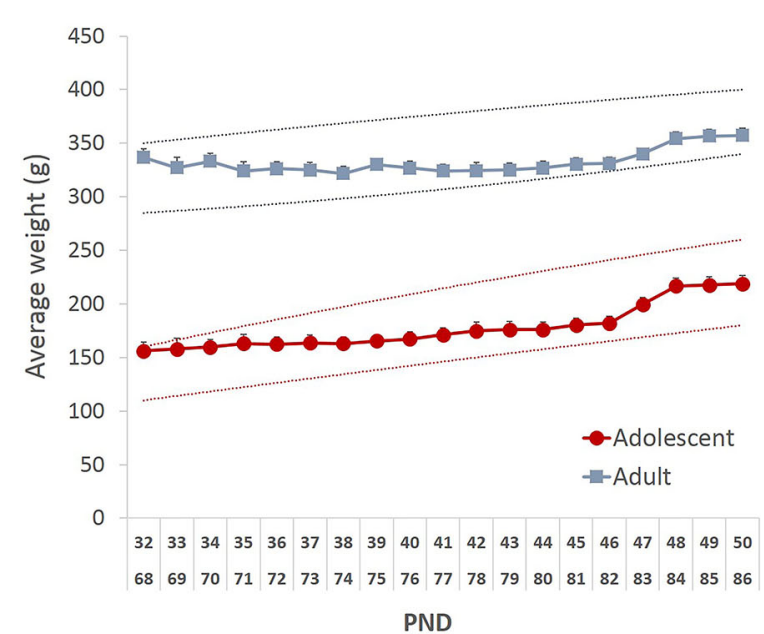

B

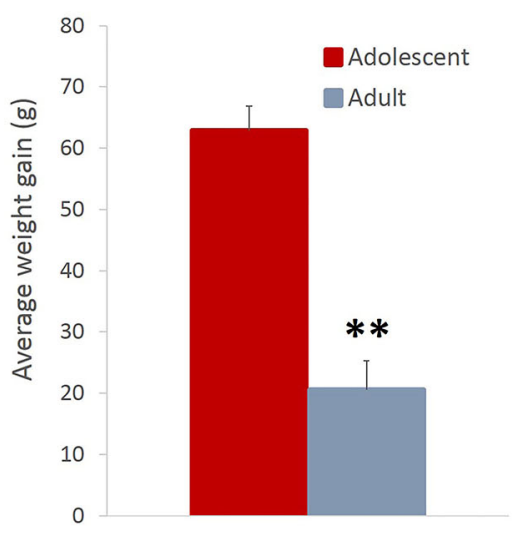

FIGURE 1 | Mild food restriction did not compromise healthy development of the animals. The weights were monitored daily from the beginning of food restriction and until the end of behavioral testing. (A) Although there was an initial weight loss in adult group (average maximal weight loss $=11 \%$ of baseline), both age groups showed an increase in weight by the end of the study. The dashed lines represent the approximate normal growth rate boundaries provided by Charles River Laboratories. (B) As expected, the average weight gain in adolescent animals was higher than in adults. Line and bar graphs represent group averages + SEM. ${ }^{\star \star} p<0.01$.

\begin{tabular}{|l|l|l|l|l|}
\hline &
\end{tabular}

showed low levels of nose-poking the screen during the ITI, and there were no differences between adult and adolescent animals (Figure 2B; $t_{(6)}=0.614, p=0.562$ ). There were no age group differences in number of food magazine entries in ITT (mean adolescent $=351$; mean adult $\left.=329.5 ; t_{(6)}=0.375, p=0.721\right)$. However, adolescent animals demonstrated increased rewardseeking behavior (mean number magazine entries $=188.54$ ) in MTT compared to adults (mean $=138.5 ; t_{(6)}=3.128, p<$ $0.05)$. Latencies to collect reward and touch the next stimulus after reward receipt have been suggested to index sensitivity to reinforcing properties of the reward. Overall, there were no differences in latency to collect reward (Figure 3A; ITT: $t_{(6)}=1.576, p=0.166$; MTT: $\left.t_{(6)}=0.919, p=0.394\right)$ or touch the image (Figure 3B; ITT: $t_{(6)}=0.726, p=0.495$; MTT: $t_{(6)}=0.958$; $p=0.375)$ between the two age groups. Although adolescent animals were faster to collect reward during the later stage of the training (Figure 3A) this difference did not reach statistical significance: no main effect of time $\left(F_{(1,6)}=1.274, p=0.302\right)$ or time $\times$ group $\left(F_{(1,6)}=3.36, p=0.117\right)$ interaction were observed. 
A

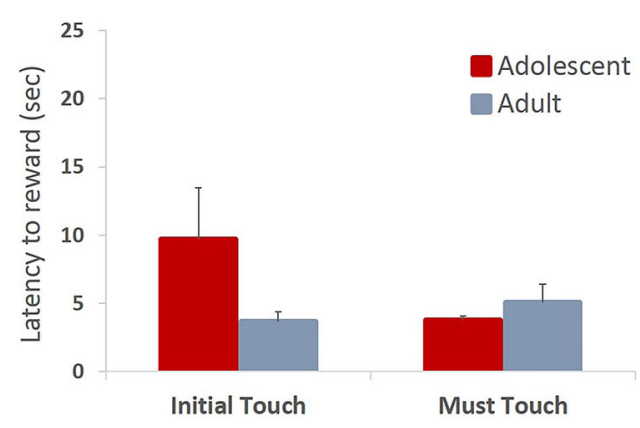

FIGURE 3 | Latencies to collect reward and respond on the subsequent trial in operant stimulus-reward association learning tasks. Bar graphs represent mean latency (sec) to collect reward following a correct response (A) or touch the stimulus on the subsequent trial $(\mathbf{B})+$ SEM. There were no
B

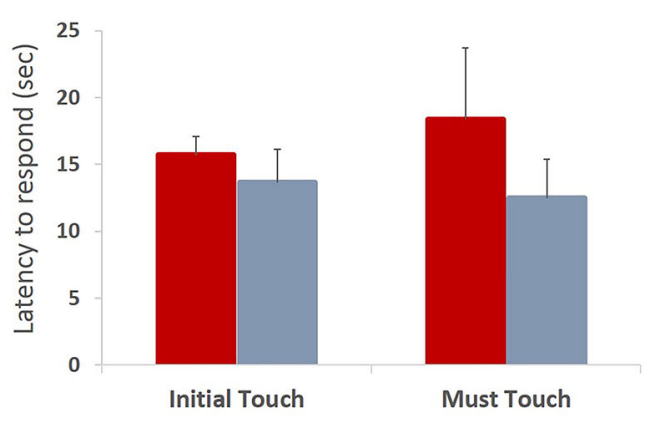

differences in latencies to collect reward or touch the stimulus between the two age groups in either of the training stages. Although adolescent animals were faster to collect reward during the later stage of the training, this difference did not reach statistical significance.
In the ITT phase of learning, animals can receive a reward of larger magnitude (HR; three sugar pellets) upon touching the stimulus, or a low reward (LR; one sugar pellet) noncontingently if they fail to touch the stimulus within allotted time. Therefore, the magnitude of the received reward could affect subsequent behavior. We hypothesized that animals would be faster to collect larger rewards respond on subsequent trials after receiving the large reward. Contrary to our hypothesis, there were no differences in latencies to collect rewards between adolescents and adults. Adolescent animals were somewhat slower to collect the HR that were contingent on their response compared to the "free" LR that was delivered at the end of the trial if the animals failed to respond, although this difference did not reach statistical significance: no main effect of trial type $\left(F_{(1,6)}=2.076, p=0.2\right)$ or trial type $\times$ age group interaction $\left(F_{(1,6)}=2.178, p=0.19\right)$ were observed (Figure 4A). Thus, the outcome of the previous trial did not have an effect on the latency to collect the reward (all $p$ values > 0.05). We did, however, observe a between-group difference in the latency to touch the stimulus depending on the outcome of the preceding trial: trial type $\times$ age group interaction $\left(F_{(1,6)}=8.105, p=0.029\right)$. Adolescent animals were faster to respond on the subsequent trial after receiving the LR compared to adult animals (Figure 4B; $p<0.05$ ). This may suggest that adolescent animals were more sensitive to receiving reward of a lesser magnitude than expected (negative contrast). It needs to be noted that in the ITT task animals responded to the stimulus in the majority of trials (mean adolescent $68 \%$, mean adult $74 \%$ ), therefore delivery of $\mathrm{HR}$ was a rule, rather than an exception. The pattern of observed responses suggests that animals respond differently to negative contrasts depending on their developmental stage. Whereas there is a tendency for invigoration of response following lesser-than-expected outcome in adolescent animals, possibly in an attempt to increase overall

\section{A}

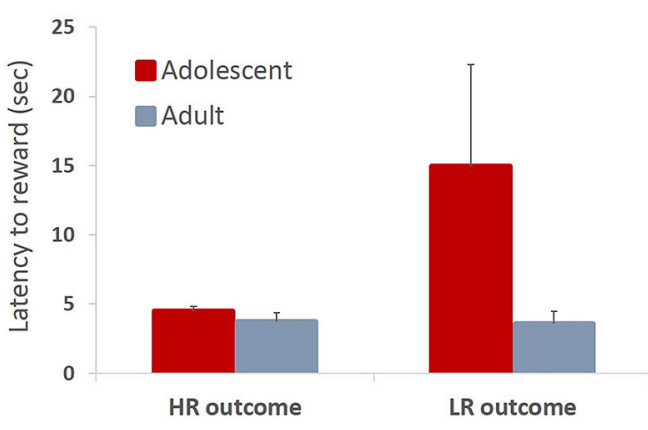

FIGURE 4 | Latencies to collect reward and respond on the subsequent trial depending on the outcome of the previous trial. Bar graphs represent mean latency (sec) + SEM. In the ITT phase of learning, animals can receive a reward of larger magnitude (HR; three sugar pellets) upon touching the stimulus, or a low reward (LR; one sugar pellet) non-contingently if they fail to touch the stimulus within allotted time. The magnitude of the received reward could affect subsequent behavior. (A) Adolescent animals were slower to collect the HR that
B

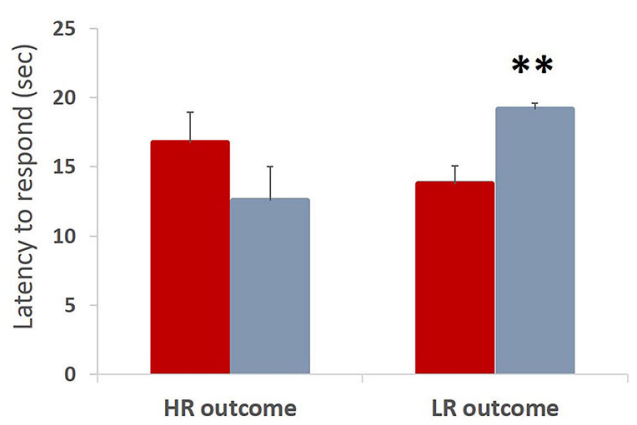

was contingent on their response compared to the "free" LR that was delivered at the end of the trial if the animals failed to respond, although this difference did not reach statistical significance. (B) Adolescent animals were significantly faster to respond on the subsequent trial after receiving the LR compared to adult animals. Whereas there was a tendency for invigoration of response following lesser-than-expected outcome in adolescent animals, adult animals were slowed by such outcomes. ${ }^{* \star} p<0.01$. 
number of rewards collected, adult animals are slowed by such outcomes.

In summary, the present results revealed a lack of developmental differences in stimulus-reward association learning: adolescent animals were as fast as adults to master the task and direct their responses toward the relevant stimuli. However, these results suggest developmentally different responses to differences in outcome values, which may manifest in a distinct pattern of choices in tasks measuring motivation to obtain rewards (Experiment 2).

\section{Experiment 2. Adolescent Behavior is Characterized by Increased Motivation to Obtain Larger Rewards Over Less Valuable Freely Available Options}

We were interested in directly comparing developmental differences in animals' motivation to obtain rewards of different magnitudes. Animals were trained on a task allowing them to choose between three available courses of action, each associated with different effort requirement and outcome values. This task is more ecologically valid and has been previously shown to effectively reveal differences in reward valuation following methamphetamine treatment, that are not easily observed in a typical T-maze effort discounting task (Stolyarova et al., 2015). In accordance with Experiment 1, there were no group differences in the number of sessions that the animals required to complete pre-training (mean adolescent $=5.63$; mean adult $=5.75)$ or reach stable performance (mean adolescent $=5.13$; mean adult $=4.75$; all $p$ values $>0.39$ ). An omnibus ANOVA revealed a significant main effect of age group on choice preferences across reward arms $\left(F_{(3,12)}=10.963, p=0.001\right.$, Wilk's $\left.\Lambda=0.267\right)$. A significant effect of age group was observed for $\operatorname{LER}\left(F_{(1,14)}=34.717\right.$, $p<0.001)$ and $\operatorname{MER}\left(F_{(1,14)}=8.262, p=0.012\right)$, but not HER
$\left(F_{(1,14)}=3.053, p=0.102\right)$ choices, with adolescent animals choosing significantly less LER and more MER (Figure 5A) compared to adult group. Adult animals distributed their choices uniformly between LER and MER goal arm options $(p=0.421)$, but chose significantly more LER $(p<0.001)$ and MER ( $p=0.005$ ) compared to HER, demonstrating reward devaluation as a function of increases in barrier heights. This trend was absent in the adolescent group, which showed a clear preference for MER over LER $(p=0.001)$ and HER $(p=0.004)$ options. When MER and HER arm choices were combined to evaluate preference for easily available over effortful options associated with greater reward magnitudes, an age group difference was observed: adolescent animals chose significantly less LER and significantly more MER and HER compared to adults $(p<0.001)$. Notably, the preference for effortful options associated with greater reward magnitudes was present in adolescents $(p<0.001)$ but not the adult group $(p=0.407)$ (Figure 5B).

Trial latencies increased with barrier height in both adolescent and adult animals (all $p$ values $<0.01$ ). Omnibus analysis revealed a significant main effect of age group on trial latencies across reward $\operatorname{arms}\left(F_{(3,12)}=6.944, p=0.007\right.$, Wilk's $\left.\Lambda=0.346\right)$. A significant effect of age group was observed for MER $\left(F_{(1,13)}=22.841, p<0.001\right)$ and $\operatorname{HER}\left(F_{(1,13)}=6.022, p=0.029\right)$, but not $\operatorname{LER}\left(F_{(1,13)}=0.224, p=0.643\right)$ choices, with adolescent animals taking significantly less time to complete MER and HER trials compared to adult group (Figure 6).

\section{Experiment 3}

Adolescent Animals Show Lower Levels of D1, but not D2 Receptor Expression in Striatum

We found non-significant increases in expression of D1 receptors in frontal cortex and significantly reduced D1 receptor

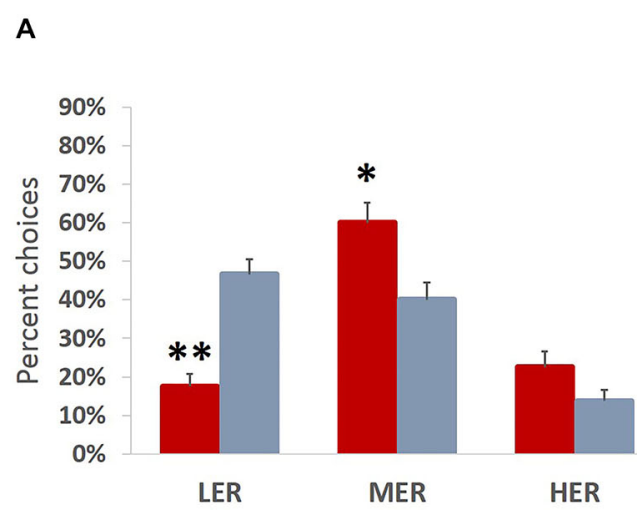

FIGURE 5 | Increased motivation to obtain larger rewards over less valuable freely available options in adolescent animals. Bar graphs represent percent high effort/reward (HER), medium effort/reward (MER), and low effort/reward (LER) choices + SEM. Animals were trained on a task allowing them to choose between three available courses of action, each associated with different effort requirement and outcome values. (A) Adolescent animals chose significantly less LER and more MER compared to the adult group. Adult animals demonstrated reward devaluation as a function of increases in barrier

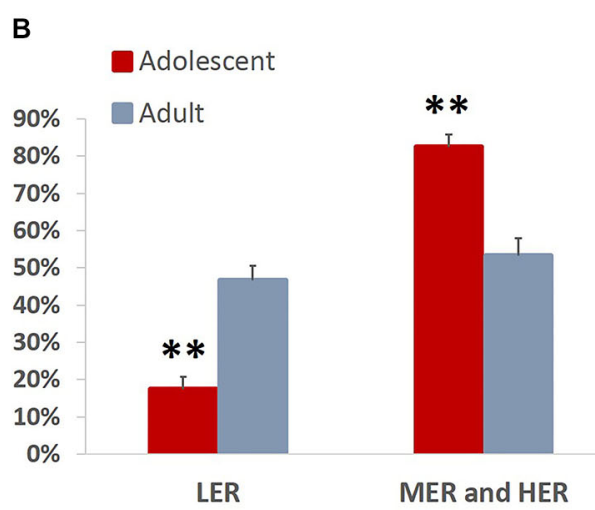

heights. This trend was absent in the adolescent group, which showed a clear preference for MER over LER and HER options. (B) MER and HER arm choices were combined to evaluate preference for easily available over effortful options associated with greater reward magnitudes. Adolescent animals chose significantly less LER and significantly more MER and HER compared to adults. Notably, the preference for effortful options associated with greater reward magnitudes over less profitable freely available options was present in the adolescent but not the adult group. ${ }^{*} p<0.05,{ }^{\star *} p<0.01$. 


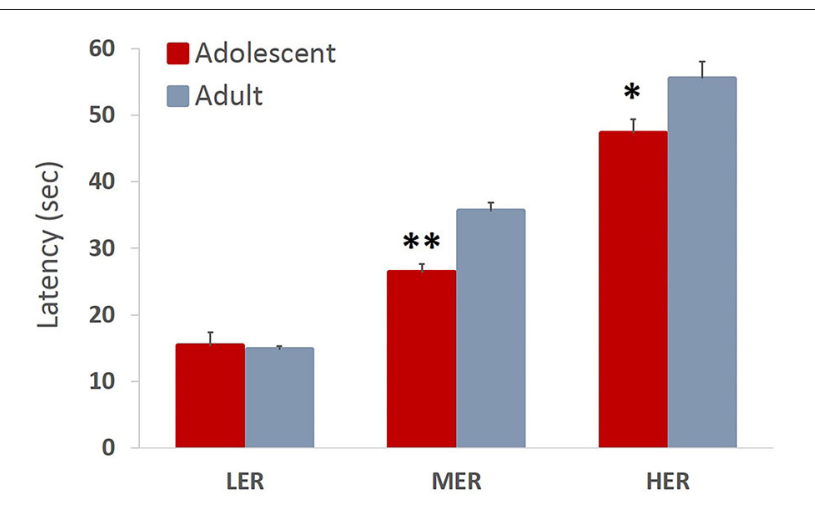

FIGURE 6 | Trial latencies in an effortful reward valuation task. Bar graphs represent average latency (sec) to complete the trial + SEM. Trial latencies increased with barrier height in both adolescent and adult animals. Adolescent animals were faster to complete MER and HER trials compared to adult group. ${ }^{*} p<0.05,{ }^{* *} p<0.01$.

expression in striatum $\left(t_{(14)}=2.204, p=0.045\right)$ of late adolescent compared to adult animals (Figure 7A). Importantly, this is consistent with previous reports which indicate that animals in early adolescence show greater levels of D1 receptors that are gradually pruned away by early adulthood (Teicher et al., 1995; Tarazi and Baldessarini, 2000). Therefore, the present results suggest that the reward experiences in adolescents may exaggerate normal pruning patterns and result in lower D1R levels as compared to the same experiences encountered in adulthood. The levels of D2 receptor expression in the striatum were not different between the animals that underwent behavioral testing during adolescence or adulthood (Figure 7B).

\section{Increased Expression of PSA-NCAM in Adolescent Animals is Associated with Greater Effort Expenditure Toward Larger Rewards}

The present study revealed increased levels of PSA-NCAM in frontal cortex $\left(t_{(13)}=3.993, p=0.002\right)$ and amygdala $\left(t_{(14)}=2.35, p=0.034\right)$ of animals tested in adolescence as compared to animals tested in adulthood (Figure 8A). Interestingly the levels of PSA-NCAM in frontal cortex, not amygdala, were strongly and positively correlated with HER reward choices when considering the entire cohort of animals $\left(r_{(16)}=0.737, p<0.01\right)$ and in adolescents alone $\left(r_{(7)}=0.909\right.$, $p<0.01)$, but not in adult animals considered independently (Figure 8B).

\section{Discussion}

Preclinical research is aimed at understanding maladaptive behaviors induced by the detrimental effects of developmental experience, specifically with drugs of abuse and stressors. Suboptimal strategies may have a distinct meaning for adolescent and adult animals, depending on the temporal proximity of the reproductive period (Gluckman et al., 2007). For example, increased energy expenditure toward palatable foods may be suboptimal in adult animals that need to invest more time and effort in searching for potential mates or providing care and shelter for offspring. Conversely, foraging for nutritional rewards is critical for adolescent animals to ensure immediate survival, promote growth and increase reproductive fitness later in life. The present findings inform our understanding of behavioral phenotypes at different developmental stages. Specifically, we show that adolescent animals are indistinguishable from adults in appetitive associative learning, but exhibit distinct pattern of responses following a receipt of lesser-than expected outcome. Increased sensitivity to differences in outcome values was further supported by an enhanced motivation to invest effort to obtain larger rewards over less valuable, freely available options. Additionally, we report distinct expression patterns of frontocortical and amygdalar PSA-NCAM and striatal dopamine receptors depending on developmental period. Importantly, only frontocortical synaptic remodeling was associated with outcome valuation.
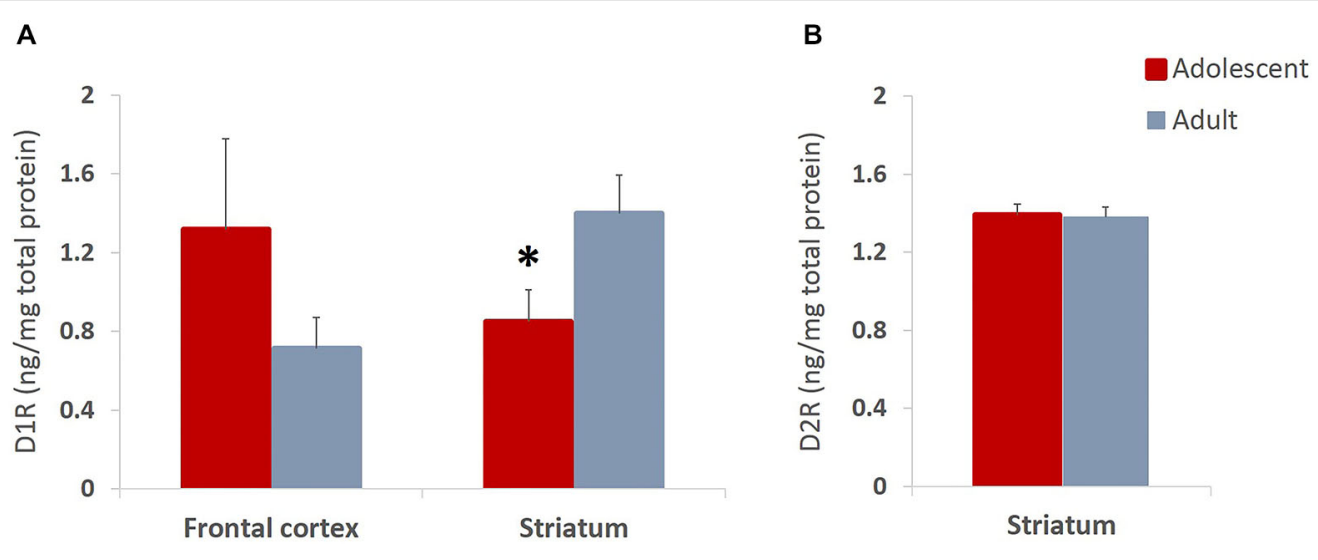

FIGURE 7 | Dopamine D1 and D2 receptor expression in adolescent and adult animals. The bar graphs represent the concentration of D1R (A) and D2R (B) protein (ng/mg of total protein) + SEM. (A) D1R expression was non-significantly increased in frontal cortex and significantly reduced in striatum of animals that had experiences with reward learning during adolescence compared to animals that went through the same learning in adulthood. (B) The levels of D2 receptor expression were not different between the animals that underwent behavioral testing during adolescence or adulthood. ${ }^{*} p<0.05$. 


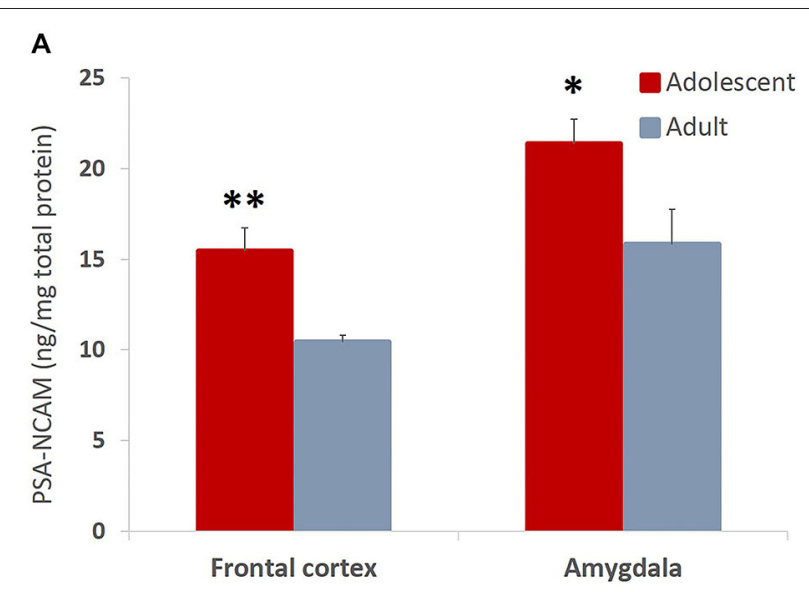

B

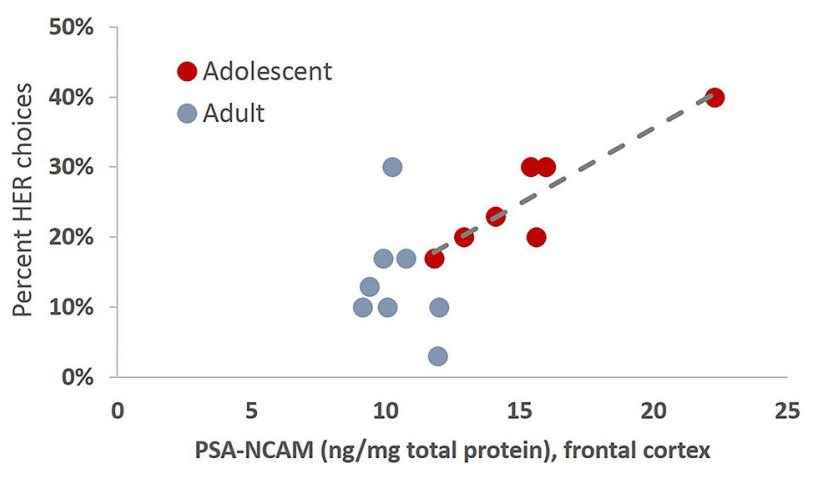

FIGURE 8 | Developmentally-specific patterns of PSA-NCAM expression are associated with greater effort expenditure toward larger rewards. (A) The bar graphs represent the concentration of PSA-NCAM ( $\mathrm{ng} / \mathrm{mg}$ of total protein) + SEM. The levels of PSA-NCAM were increased in frontal cortex and amygdala in late adolescent compared to adult animals that had been trained on an effortful reward valuation task. (B) PSA-NCAM levels in PFC were strongly and positively associated with HER choices when considering the entire cohort of animals and in adolescents alone, but not in adult animals considered independently. The regression line is given for the adolescent group only ${ }^{*} p<0.05,{ }^{* *} p<0.01$.

\section{Reward Learning is Similar in Adolescent and Adult Animals}

Associative learning is highly important for many characteristic animal behaviors in the wild, including exploration of novelty, increased attention to change, and approach to potential rewards (Cloninger and Gilligan, 1987). An ability to learn the association between the appetitive outcomes and predictive stimuli provides an evolutionary advantage as it allows animals to maximize the rewards, of great importance to mammalian species (Bitterman, 1975). The results of the present investigation suggest that by the time rodents transition from complete reliance on their adult conspecifics and begin exploring their surroundings independently (i.e., adolescent period; Galef, 1981), they already possess associate reward learning skills. We observed no age differences in stimulus-reward and instrumental learning: adolescent animals were as fast as adults to master the operant task and direct their responses toward relevant stimuli. Similarly, they efficiently learned the spatial distribution of reward densities in the maze task and established stable choice behavior at a rate comparable to adults.

Although we did not examine reward learning earlier in development, the present data suggest that appetitive learning is established before adolescence. It needs to be noted, however, that one previous report demonstrated impaired odor-discrimination learning in adolescent compared to both juvenile and adult animals (Garske et al., 2013). In that task, which may be more ethologically-relevant for rodents than our visual task, animals were first trained to dig in a cup filled with unscented playground sand to obtain a palatable food reward, after which they were presented with two odorized cups only one of which contained the reward. Adolescent animals were slower to acquire this odor-association task, an effect that disappeared with pretraining during the juvenile period. Taken together, these results suggest that adolescent animals are not different on measures of simple appetitive reward learning; they were still able to acquire sand-reward association. However, rats displayed a limited ability to fine-tune cue representations and demonstrate learning difficulties when cues had more than one attribute. Previous reports also indicate compromised ability to behaviorally adapt to a change in operant contingencies and extinguish previously reinforced responding in adolescent animals (Sturman et al., 2010; Andrzejewski et al., 2011). However, similar to the present results, younger animals in both of these studies efficiently learned simple stimulus- and actionreward associations.

\section{Adolescent and Adult Animals Display Distinct Patterns of Responses to Outcome Values, which Manifest in Increased Effort Toward More Profitable Options}

The only developmental differences observed in the operant task were in reward-seeking behavior and the response strategy following a receipt of lesser-than expected outcome. Adolescent animals were faster to respond on the subsequent trial after receiving a small reward, which happened in the minority of the trials, suggesting increased sensitivity to a violation of reward expectancy. Indeed, younger animals may be evolutionarily primed to be more sensitive to changes in their environment, as it allows to increase overall immediate profitability of the situation and prepare themselves for the future (Spear, 2000; Gluckman et al., 2007). Whereas there was a strong tendency for invigoration of response following lesser-than-expected outcomes in adolescent animals (possibly in an attempt to increase overall number of rewards collected), adult animals were slowed by such outcomes.

In accordance with their increased sensitivity to differences in outcome value, adolescent animals also displayed increased motivation to work for rewards of greater magnitude. Their choice behavior was characterized by increased effort expenditure toward larger rewards, while adult animals showed a clear pattern of reward devaluation as a function 
of increased barrier heights. The observed differences in choice preferences may be due to potentiated reactions to novel palatable foods in younger animals. Adolescents have been previously shown to display conditioned place preference, a measure of reward, to novelty; an effect that is absent in adults (Douglas et al., 2003). Additionally, adolescent animals are more sensitive to natural rewards, consume more sucrose solution and exhibit greater positive taste responses than their adult counterparts (Wilmouth and Spear, 2009). Alternatively, adolescents may be more sensitive to changes in unpredictable conditions in their habitat, which modulates effort expenditure (McNamara et al., 2013). Specifically, in the present study, both adult and adolescent animals were raised in a benign, nutritionally optimal environment, with food and water provided ad libitum, and were socially housed; they did not need to actively forage for rewards. The mismatch that was introduced as a result of short-term food restriction, may have had a more profound impact on adolescents compared to adults. These findings suggest that experiences during adolescence may have more potential adaptive significance than those encountered later in adulthood.

\section{Neurodevelopmental Correlates of Reward-Sensitive Endophenotype}

Dopaminergic neurotransmission within the striatum has long been recognized as critical for incentive motivation and optimal response allocation to rewards (Berridge and Robinson, 1998; Salamone and Correa, 2002; Ostlund et al., 2011; Salamone et al., 2012; Richard et al., 2013). D1R and $\mathrm{D} 2 \mathrm{R}$ density in the striatum peaks at the onset of the adolescent period, followed by extensive pruning to adult levels (Gelbard et al., 1989; Teicher et al., 1995; Tarazi and Baldessarini, 2000). The results of the present investigation revealed unaltered D2R expression, but reduced D1R expression in the striatum of animals that had experiences with reward learning during adolescence compared to animals that went through the same learning in adulthood. Decreased expression of D1R may result in diminished neuronal excitability in the striatonigral pathway upon dopamine release (Aosaki et al., 1998), and may ultimately lead to reduced learning from positive outcomes (Cox et al., 2015). It needs to be noted that because the brains were collected following training and establishment of stable performance, we are unable to distinguish age-specific from experience-dependent receptor expression profiles. However, previous reports indicate that D1R expression reaches mature levels by early adulthood (Teicher et al., 1995; Tarazi and Baldessarini, 2000). Therefore, reward experiences during adolescence may exaggerate normal pruning patterns and result in lower D1R levels as compared to the same experiences encountered in adulthood. Increased D1R expression early in adolescence (Gelbard et al., 1989; Tarazi and Baldessarini, 2000) may aid in establishing a pattern of behavior characterized by greater effort expenditure toward larger rewards, whereas decreased levels of D1R expression at the onset of adulthood would render animals less vulnerable to the effects of experiences with potent reinforcers.

Information transfer between amygdala and PFC has been shown to be critical for optimal reward-driven effort expenditure in maze tasks (Floresco and Ghods-Sharifi, 2007), with basolateral amygdala (BLA) signaling differences in reward magnitude (Salinas et al., 1993; Pratt and Mizumori, 1998). Cortical projections from BLA undergo remarkable development during adolescence (Casey et al., 2000; Cunningham et al., 2002, 2008; Brenhouse and Andersen, 2011). PSA-NCAM may play an important role in such structural and functional changes given its importance in activity-dependent synaptic remodeling and developmental events (Muller et al., 1996, 2010; Dey et al., 1999; Durbec and Cremer, 2001; Kiss and Muller, 2001; Welzl and Stork, 2003) resulting in prominent patterns of expression in regions undergoing active functional restructuring (Nacher et al., 2002a,b; Seki, 2002; Varea et al., 2005). Tsoory et al. (2008) reported significant decreases in PSA-NCAM expression with developmental progression from adolescence into adulthood in amygdala and hippocampus of naïve animals. The results of the present investigation revealed increased levels of PSA-NCAM expression in PFC and amygdala in late adolescent compared to adult animals that had been trained on an effortful reward valuation task. Intriguingly, PSANCAM levels in PFC were strongly and positively associated with HER choices in adolescents, but not in adult animals. To our knowledge, this is the first report showing a link between outcome valuation and developmentally-specific differences in PSA-NCAM expression. PSA-NCAM in the adult brain is restricted to interneurons, at least in PFC and BLA, and may aid in the incorporation of interneurons into circuitry to modulate local inhibition (Gascon et al., 2007; Gómez-Climent et al., 2011; Nacher et al., 2013). Increased levels of PSA-NCAM expression in adolescent animals in the present study may index increased structural plasticity within these brain regions and represent a neural correlate of a reward-sensitive endophenotype. However, additional investigations utilizing direct manipulations targeted to adolescent BLA and subregions within PFC are needed to establish a causal role for PSA-NCAM in adolescent-specific behavioral traits.

\section{Author Contributions}

AS and AI designed research, analyzed data and wrote the paper; AS performed research.

\section{Acknowledgments}

This work was supported by UCLA's Division of Life Sciences Recruitment and Retention fund (Izquierdo). We also thank Adrianna De La Torre and Amador Bugarin for help with collecting the operant learning data (Experiment 1), as well as Andrew B. Thompson, Eric Harvey, and Amandeep Kaur for help with tissue processing and ELISA assays (Experiment 3). 


\section{References}

Andrzejewski, M. E., Schochet, T. L., Feit, E. C., Harris, R., McKee, B. L., and Kelley, A. E. (2011). A comparison of adult and adolescent rat behavior in operant learning, extinction and behavioral inhibition paradigms. Behav. Neurosci. 125, 93-105. doi: 10.1037/a0022038

Aosaki, T., Kiuchi, K., and Kawaguchi, Y. (1998). Dopamine D1-like receptor activation excites rat striatal large aspiny neurons in vitro. J. Neurosci. 18, 5180-5190.

Baldwin, A. E., Sadeghian, K., and Kelley, A. E. (2002). Appetitive instrumental learning requires coincident activation of NMDA and dopamine D1 receptors within the medial prefrontal cortex. J. Neurosci. 22, 1063-1071.

Barker, J. M., Torregrossa, M. M., and Taylor, J. R. (2012). Low prefrontal PSA-NCAM confers risk for alcoholism-related behavior. Nat. Neurosci. 15, 1356-1358. doi: 10.1038/nn.3194

Baxter, M. G., Parker, A., Lindner, C. C., Izquierdo, A. D., and Murray, E. A. (2000). Control of response selection by reinforcer value requires interaction of amygdala and orbital prefrontal cortex. J. Neurosci. 20, 4311-4319.

Beninger, R. J., and Miller, R. (1998). Dopamine D1-like receptors and rewardrelated incentive learning. Neurosci. Biobehav. Rev. 22, 335-345. doi: 10. 1016/s0149-7634(97)00019-5

Berridge, K. C., and Robinson, T. E. (1998). What is the role of dopamine in reward: hedonic impact, reward learning, or incentive salience? Brain Res. Brain Res. Rev. 28, 309-369. doi: 10.1016/s0165-0173(98)00019-8

Bisaz, R., Conboy, L., and Sandi, C. (2009). Learning under stress: a role for the neural cell adhesion molecule NCAM. Neurobiol. Learn. Mem. 91, 333-342. doi: $10.1016 /$ j.nlm.2008.11.003

Bitterman, M. E. (1975). The comparative analysis of learning. Science 188, 699-709. doi: 10.1126/science.188.4189.699

Blair, K., Marsh, A. A., Morton, J., Vythilingam, M., Jones, M., Mondillo, K., et al. (2006). Choosing the lesser of two evils, the better of two goods: specifying the roles of ventromedial prefrontal cortex and dorsal anterior cingulate in object choice. J. Neurosci. 26, 11379-11386. doi: 10.1523/jneurosci.164006.2006

Brenhouse, H. C., and Andersen, S. L. (2011). Developmental trajectories during adolescence in males and females: a cross-species understanding of underlying brain changes. Neurosci. Biobehav. Rev. 35, 1687-1703. doi: 10. 1016/j.neubiorev.2011.04.013

Cagniard, B., Beeler, J. A., Britt, J. P., McGehee, D. S., Marinelli, M., and Zhuang, X. (2006). Dopamine scales performance in the absence of new learning. Neuron 51, 541-547. doi: 10.3410/f.1040616.489625

Calandreau, L., Márquez, C., Bisaz, R., Fantin, M., and Sandi, C. (2010). Differential impact of polysialyltransferase ST8SiaII and ST8SiaIV knockout on social interaction and aggression. Genes Brain Behavior. 9, 958-967. doi: 10. 1111/j.1601-183x.2010.00635.x

Casey, B. J., Giedd, J. N., and Thomas, K. M. (2000). Structural and functional brain development and its relation to cognitive development. Biol. Psychol. 54, 241-257. doi: 10.1016/s0301-0511(00)00058-2

Castillo-Gómez, E., Gómez-Climent, M. A., Varea, E., Guirado, R., Blasco-Ibáñez, J. M., Crespo, C., et al. (2008). Dopamine acting through D2 receptors modulates the expression of PSA-NCAM, a molecule related to neuronal structural plasticity, in the medial prefrontal cortex of adult rats. Exp. Neurol. 214, 97-111. doi: 10.1016/j.expneurol.2008.07.018

Cloninger, C. R., and Gilligan, S. B. (1987). Neurogenetic mechanisms of learning: a phylogenetic perspective. J. Psychiatr. Res. 21, 457-472. doi: 10.1016/00223956(87)90094-x

Cordero, M. I., Rodríguez, J. J., Davies, H. A., Peddie, C. J., Sandi, C., and Stewart, M. G. (2005). Chronic restraint stress down-regulates amygdaloid expression of polysialylated neural cell adhesion molecule. Neuroscience 133, 903-910. doi: $10.1016 /$ j.neuroscience.2005.03.046

Cox, S. M., Frank, M. J., Larcher, K., Fellows, L. K., Clark, C. A., Leyton, M., et al. (2015). Striatal D1 and D2 signaling differentially predict learning from positive and negative outcomes. Neuroimage 109, 95-101. doi: 10.1016/j.neuroimage. 2014.12.070

Cunningham, M. G., Bhattacharyya, S., and Benes, F. M. (2002). Amygdalocortical sprouting continues into early adulthood: implications for the development of normal and abnormal function during adolescence. J. Comp. Neurol. 453, 116-130. doi: 10.1002/cne.10376
Cunningham, M. G., Bhattacharyya, S., and Benes, F. M. (2008). Increasing interaction of amygdalar afferents with GABAergic interneurons between birth and adulthood. Cereb. Cortex 18, 1529-1535. doi: 10.1093/cercor/bhm183

Dey, P. M., Gochfeld, M., and Reuhl, K. R. (1999). Developmental methylmercury administration alters cerebellar PSA-NCAM expression and Golgi sialyltransferase activity. Brain Res. 845, 139-151. doi: 10.1016/s00068993(99)01887-9

Douglas, L. A., Varlinskaya, E. I., and Spear, L. P. (2003). Novel-object place conditioning in adolescent and adult male and female rats: effects of social isolation. Physiol. Behav. 80, 317-325. doi: 10.1016/j.physbeh.2003. 08.003

Durbec, P., and Cremer, H. (2001). Revisiting the function of PSA-NCAM in the nervous system. Mol. Neurobiol. 24, 53-64. doi: 10.1385/mn:24:1-3:053

Floresco, S. B., and Ghods-Sharifi, S. (2007). Amygdala-prefrontal cortical circuitry regulates effort-based decision making. Cereb. Cortex 17, 251-260. doi: $10.1093 /$ cercor/bhj143

Galef, B. G. Jr. (1981). "The ecology of weaning: parasitism and the achievement of independence by altricial mammals," in Parental Care in Mammals, eds D. J. Gubernick and P. H. Klopfer (New York, NY: Plenum Press), 211-241.

Garske, A. K., Lawyer, C. R., Peterson, B. M., and Illig, K. R. (2013). Adolescent changes in dopamine D1 receptor expression in orbitofrontal cortex and piriform cortex accompany an associative learning deficit. PLoS One 8:e56191. doi: 10.1371/journal.pone.0056191

Gascon, E., Vutskits, L., and Kiss, J. Z. (2007). Polysialic acid-neural cell adhesion molecule in brain plasticity: from synapses to integration of new neurons. Brain Res. Rev. 56, 101-118. doi: 10.1016/j.brainresrev.2007.05.014

Gelbard, H. A., Teicher, M. H., Faedda, G., and Baldessarini, R. J. (1989). Postnatal development of dopamine D1 and D2 receptor sites in rat striatum. Brain Res. Dev. Brain Res. 49, 123-130. doi: 10.1016/0165-3806(89)90065-5

Gluckman, P. D., Hanson, M. A., and Beedle, A. S. (2007). Early life events and their consequences for later disease: a life history and evolutionary perspective. Am. J. Hum. Biol. 19, 1-19. doi: 10.1002/ajhb.20590

Gómez-Climent, M. Á., Guirado, R., Castillo-Gómez, E., Varea, E., GutierrezMecinas, M., Gilabert-Juan, J., et al. (2011). The polysialylated form of the neural cell adhesion molecule (PSA-NCAM) is expressed in a subpopulation of mature cortical interneurons characterized by reduced structural features and connectivity. Cereb. Cortex 21, 1028-1041. doi: 10.1093/cercor/ bhq177

Groman, S. M., Lee, B., London, E. D., Mandelkern, M. A., James, A. S., Feiler, K., et al. (2011). Dorsal striatal D2-like receptor availability covaries with sensitivity to positive reinforcement during discrimination learning. J. Neurosci. 31, 7291-7299. doi: 10.1523/jneurosci.0363-11.2011

Hildebrandt, H., Mühlenhoff, M., Oltmann-Norden, I., Röckle, I., Burkhardt, H., Weinhold, B., et al. (2009). Imbalance of neural cell adhesion molecule and polysialyltransferase alleles causes defective brain connectivity. Brain 132, 2831-2838. doi: 10.1093/brain/awp117

Izquierdo, A., Belcher, A. M., Scott, L., Cazares, V. A., Chen, J., O’Dell, S. J., et al. (2010). Reversal-specific learning impairments after a binge regimen of methamphetamine in rats: possible involvement of striatal dopamine. Neuropsychopharmacology 35, 505-514. doi: 10.1038/npp.2009.155

Kelley, A. E., Schochet, T., and Landry, C. F. (2004). Risk taking and novelty seeking in adolescence: introduction to part I. Ann. N Y Acad. Sci. 1021, 27-32. doi: 10.1196/annals.1308.003

Kiss, J. Z., and Muller, D. (2001). Contribution of the neural cell adhesion molecule to neuronal and synaptic plasticity. Rev. Neurosci. 12, 297-310. doi: 10.1515/revneuro.2001.12.4.297

Kochlamazashvili, G., Senkov, O., Grebenyuk, S., Robinson, C., Xiao, M. F., Stummeyer, K., et al. (2010). Neural cell adhesion molecule-associated polysialic acid regulates synaptic plasticity and learning by restraining the signaling through GluN2B-containing NMDA receptors. J. Neurosci. 30, 4171-4183. doi: 10.1523/jneurosci.5806-09.2010

Kosheleff, A. R., Rodriguez, D., O’Dell, S. J., Marshall, J. F., and Izquierdo, A. (2012). Comparison of single-dose and extended methamphetamine administration on reversal learning in rats. Psychopharmacology (Berl) 224, 459-467. doi: 10.1007/s00213-012-2774-1

Laviola, G., Macrì, S., Morley-Fletcher, S., and Adriani, W. (2003). Risktaking behavior in adolescent mice: psychobiological determinants and early 
epigenetic influence. Neurosci. Biobehav. Rev. 27, 19-31. doi: 10.1016/s01497634(03)00006-x

Lopez-Fernandez, M. A., Montaron, M. F., Varea, E., Rougon, G., Venero, C., Abrous, D. N., et al. (2007). Upregulation of polysialylated neural cell adhesion molecule in the dorsal hippocampus after contextual fear conditioning is involved in long-term memory formation. J. Neurosci. 27, 4552-4561. doi: 10. 1523/jneurosci.0396-07.2007

Marco, E. M., Adriani, W., Ruocco, L. A., Canese, R., Sadile, A. G., and Laviola, G. (2011). Neurobehavioral adaptations to methylphenidate: the issue of early adolescent exposure. Neurosci. Biobehav. Rev. 35, 1722-1739. doi: 10.1016/j. neubiorev.2011.02.011

McNamara, J. M., Fawcett, T. W., and Houston, A. I. (2013). An adaptive response to uncertainty generates positive and negative contrast effects. Science 340, 1084-1086. doi: 10.1126/science.1230599

Muller, D., Mendez, P., Deroo, M., Klauser, P., Steen, S., and Poglia, L. (2010). Role of NCAM in spine dynamics and synaptogenesis. Adv. Exp. Med. Biol. 663, 245-256. doi: 10.1007/978-1-4419-1170-4_16

Muller, D., Wang, C., Skibo, G., Toni, N., Cremer, H., Calaora, V., et al. (1996). PSA-NCAM is required for activity-induced synaptic plasticity. Neuron 17, 413-422. doi: 10.1016/s0896-6273(00)80174-9

Nacher, J., Blasco-Ibáñez, J. M., and McEwen, B. S. (2002a). Non-granule PSANCAM immunoreactive neurons in the rat hippocampus. Brain Res. 930, 1-11. doi: 10.1016/s0006-8993(01)03365-0

Nacher, J., Guirado, R., and Castillo-Gómez, E. (2013). Structural plasticity of interneurons in the adult brain: role of PSA-NCAM and implications for psychiatric disorders. Neurochem. Res. 38, 1122-1133. doi: 10.1007/s11064013-0977-4

Nacher, J., Lanuza, E., and McEwen, B. S. (2002b). Distribution of PSA-NCAM expression in the amygdala of the adult rat. Neuroscience 113, 479-484. doi: 10. 1016/s0306-4522(02)00219-1

Ostlund, S. B., Wassum, K. M., Murphy, N. P., Balleine, B. W., and Maidment, N. T. (2011). Extracellular dopamine levels in striatal subregions track shifts in motivation and response cost during instrumental conditioning. J. Neurosci. 31, 200-207. doi: 10.3410/f.7805956.8200054

Pham, K., Nacher, J., Hof, P. R., and McEwen, B. S. (2003). Repeated restraint stress suppresses neurogenesis and induces biphasic PSA-NCAM expression in the adult rat dentate gyrus. Eur. J. Neurosci. 17, 879-886. doi: 10.1046/j.14609568.2003.02513.x

Pratt, W. E., and Mizumori, S. J. (1998). Characteristics of basolateral amygdala neuronal firing on a spatial memory task involving differential reward. Behav. Neurosci. 112, 554-570. doi: 10.1037/0735-7044.112.3.554

Richard, J. M., Castro, D. C., Difeliceantonio, A. G., Robinson, M. J., and Berridge, K. C. (2013). Mapping brain circuits of reward and motivation: in the footsteps of Ann Kelley. Neurosci. Biobehav. Rev. 37(9 Pt. A), 1919-1931. doi: 10.1016/j. neubiorev.2012.12.008

Salamone, J. D., and Correa, M. (2002). Motivational views of reinforcement: implications for understanding the behavioral functions of nucleus accumbens dopamine. Behav. Brain Res. 137, 3-25. doi: 10.1016/s0166-4328(02) 00282-6

Salamone, J. D., Correa, M., Nunes, E. J., Randall, P. A., and Pardo, M. (2012). The behavioral pharmacology of effort-related choice behavior: dopamine, adenosine and beyond. J. Exp. Anal. Behav. 97, 125-146. doi: 10.1901/jeab.2012. 97-125

Salinas, J. A., Packard, M. G., and McGaugh, J. L. (1993). Amygdala modulates memory for changes in reward magnitude: reversible post-training inactivation with lidocaine attenuates the response to a reduction in reward. Behav. Brain Res. 59, 153-159. doi: 10.1016/0166-4328(93)90162-j

Schweimer, J., and Hauber, W. (2006). Dopamine D1 receptors in the anterior cingulate cortex regulate effort-based decision making. Learn. Mem. 13, 777-782. doi: $10.1101 / \mathrm{lm} .409306$

Seki, T. (2002). Expression patterns of immature neuronal markers PSA-NCAM, CRMP-4 and NeuroD in the hippocampus of young adult and aged rodents. J. Neurosci. Res. 70, 327-334. doi: 10.1002/jnr.10387
Senkov, O., Sun, M., Weinhold, B., Gerardy-Schahn, R., Schachner, M., and Dityatev, A. (2006). Polysialylated neural cell adhesion molecule is involved in induction of long-term potentiation and memory acquisition and consolidation in a fear-conditioning paradigm. J. Neurosci. 26, 10888-109898. doi: 10.1523/jneurosci.0878-06.2006

Spear, L. (2000). The adolescent brain and age-related behavioral manifestations. Neurosci. Biobehav. Rev. 24, 417-463. doi: 10.1016/S0149-7634(00)00014-2

Stolyarova, A., Thompson, A. B., Barrientos, R. M., and Izquierdo, A. (2015). Reductions in frontocortical cytokine levels are associated with long-lasting alterations in reward valuation after methamphetamine. Neuropsychopharmacology 40, 1234-1242. doi: 10.1038/npp.2014.309

Sturman, D. A., Mandell, D. R., and Moghaddam, B. (2010). Adolescents exhibit behavioral differences from adults during instrumental learning and extinction. Behav. Neurosci. 124, 16-25. doi: 10.1037/a0018463

Tarazi, F. I., and Baldessarini, R. J. (2000). Comparative postnatal development of dopamine $\mathrm{D}(1), \mathrm{D}(2)$ and $\mathrm{D}(4)$ receptors in rat forebrain. Int. J. Dev. Neurosci. 18, 29-37. doi: 10.1016/s0736-5748(99)00108-2

Teicher, M. H., Andersen, S. L., and Hostetter, J. C. Jr. (1995). Evidence for dopamine receptor pruning between adolescence and adulthood in striatum but not nucleus accumbens. Brain Res. Dev. Brain Res. 89, 167-172. doi: 10. 1016/0165-3806(95)00109-q

Trifilieff, P., Feng, B., Urizar, E., Winiger, V., Ward, R. D., Taylor, K. M., et al. (2013). Increasing dopamine D2 receptor expression in the adult nucleus accumbens enhances motivation. Mol. Psychiatry 18, 1025-1033. doi: 10. 1038/mp.2013.57

Tsoory, M., Guterman, A., and Richter-Levin, G. (2008). Exposure to stressors during juvenility disrupts development-related alterations in the PSA-NCAM to NCAM expression ratio: potential relevance for mood and anxiety disorders. Neuropsychopharmacology 33, 378-393. doi: 10.1038/sj.npp.1301397

Varea, E., Nácher, J., Blasco-Ibáñez, J. M., Gómez-Climent, M. A., Castillo-Gómez, E., Crespo, C., et al. (2005). PSA-NCAM expression in the rat medial prefrontal cortex. Neuroscience 136, 435-443. doi: 10.1016/j.neuroscience.2005.08.009

Vutskits, L., Djebbara-Hannas, Z., Zhang, H., Paccaud, J. P., Durbec, P., Rougon, G., et al. (2001). PSA-NCAM modulates BDNF-dependent survival and differentiation of cortical neurons. Eur. J. Neurosci. 13, 1391-1402. doi: 10. 1046/j.0953-816x.2001.01516.x

Walton, M. E., Bannerman, D. M., and Rushworth, M. F. (2002). The role of rat medial frontal cortex in effort-based decision making. J. Neurosci. 22, 10996-11003.

Waraczynski, M. A. (2006). The central extended amygdala network as a proposed circuit underlying reward valuation. Neurosci. Biobehav. Rev. 30, 472-496. doi: 10.1016/j.neubiorev.2005.09.001

Welzl, H., and Stork, O. (2003). Cell adhesion molecules: key players in memory consolidation? News Physiol. Sci. 18, 147-150. doi: 10.1152/nips.01 422.2002

Wilmouth, C. E., and Spear, L. P. (2009). Hedonic sensitivity in adolescent and adult rats: taste reactivity and voluntary sucrose consumption. Pharmacol. Biochem. Behav. 92, 566-573. doi: 10.1016/j.pbb.2009.02.009

Xiao, M. F., Xu, J. C., Tereshchenko, Y., Novak, D., Schachner, M., and Kleene, R. (2009). Neural cell adhesion molecule modulates dopaminergic signaling and behavior by regulating dopamine D2 receptor internalization. J. Neurosci. 29, 14752-14763. doi: 10.1523/jneurosci.4860-09.2009

Conflict of Interest Statement: The authors declare that the research was conducted in the absence of any commercial or financial relationships that could be construed as a potential conflict of interest.

Copyright (c) 2015 Stolyarova and Izquierdo. This is an open-access article distributed under the terms of the Creative Commons Attribution License (CC BY). The use, distribution and reproduction in other forums is permitted, provided the original author(s) or licensor are credited and that the original publication in this journal is cited, in accordance with accepted academic practice. No use, distribution or reproduction is permitted which does not comply with these terms. 